\title{
10 minutes of no flow: is it futile resuscitation?
}

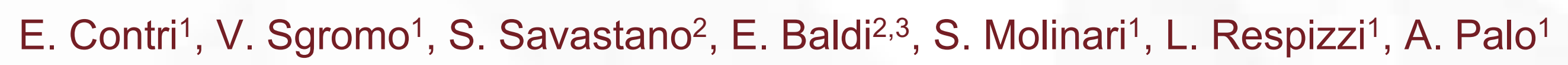

${ }^{1}$ AREU - Azienda Regionale Emergenza Urgenza - AAT Pavia, IRCCS Policlinico San Matteo Hospital, Pavia, Italy; ${ }^{2}$ Divisione di Cardiologia, IRCCS Policlinico San Matteo, Pavia, Italy ${ }^{3}$ School of Cardiovascular Disease, University of Pavia, Pavia, Italy

\section{Introduction}

The decision to start or withdraw resuscitation is very challenging in out-of-hospital cardiac arrest setting. The lack of medical records and the inability of the patient to decide for himself confers ALS team the duty to offer to the patients every realistic chance avoiding futile treatments. At this moment, without clear sign of death, rescue teams are used to start CPR as soon as possible postponing every question about the appropriateness of resuscitation. Despite the obvious sense of this practice, based on the positive correlation between the prompt start of CPR and the outcome, futile resuscitation should be avoided in respect of the patient and his family. In this brief retrospective register-based study we looked for a "no flow time" that could predict "futile" attempt resuscitation.

\section{Methods}

Using data from PaviaCARE registry, we considered all the patients with witnessed or unwitnessed cardiac arrest, with presumed or certain cardiac origin, with no bystander-CPR and in which resuscitation was attempted by EMS personnel, in the period from January $1^{\text {st }} 2016$ to April 29th 2018 . We then restricted our research to the cases with a time from call to arrival of the first EMS vehicle greater or equal than 10 minutes and we evaluated the survival rate.

\section{Results}

We found 248 eligible patients. Of these, 215 patients died on the scene, 25 patients were transported during CPR and died in the Emergency Department and only 8 patients were transported after ROSC but died before ICU discharge (from 0 to 3 days in ICU).

\section{Conclusion}

Our data show that a no-flow time of 10 minutes is enough to predict $0 \%$ survival rate so, when the time of cardiac arrest is clear and CPR not initiated by bystander, it could be used in a comprehensive evaluation to decide when withdrawn resuscitation.
Population served by EMS

\section{$=547.251$}

Out-of-hospital cardiac arrest attented
$=1560$

$$
=1560
$$

Witnessed or unwitnessed at 112 call
Cardiac or presumed cardiac origin No bystandar CPR

Resuscitation attempted by EMS

$=\mathbf{4 7 9}$ patients

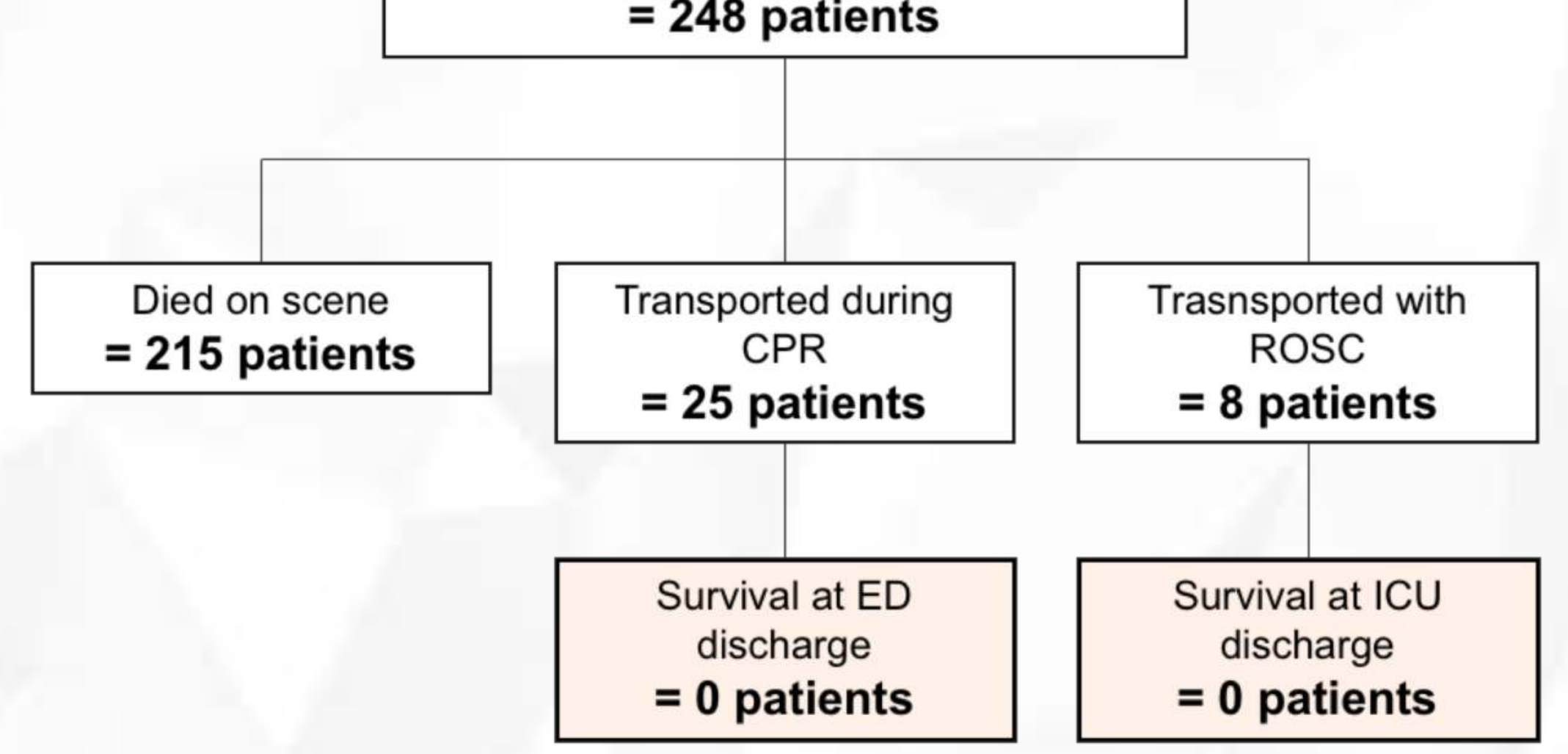

